\title{
Similar Construction Method of the Boundary Value Problem of Composite Spherical Bessel Equations
}

\author{
Qiang Wang ${ }^{1, *}$, Shunchu $\mathrm{Li}^{1}$, Ming Hu${ }^{1}$, Dongdong Gui ${ }^{2}$ \\ ${ }^{1}$ Institute of Applied Mathematics, Xihua University, Chengdu, China \\ ${ }^{2}$ Beijing Dong run ke Petroleum Technology Co,Ltd, Beijing, China \\ ${ }^{*}$ Corresponding author: wangqian1205@foxmail.com
}

Received February 27, 2015; Revised March 05, 2015; Accepted March 10, 2015

\begin{abstract}
Aimed at the boundary value problem of composite spherical Bessel equations. Through introducing similar kernel function in the left (right) region, the similar structure of solution was obtained. By analyzing the structure of solution, the solution in the left region was assembled by coefficient of left boundary value condition and similar kernel function in the left region, the solution in the right region was assembled by similar kernel function in the left (right) region; coefficient of left boundary value condition; left guide functions and coefficient of joint conditions. Thus a simple method of solving the boundary value problem of composite spherical Bessel equations is put forward, namely similar construction method; This method provides a way for solving boundary value problem of composite differential equations.
\end{abstract}

Keywords: boundary value problem, composite spherical Bessel equations, similar structure, similar kernel function

Cite This Article: Qiang Wang, Shunchu Li, Ming Hu, and Dongdong Gui, "Similar Construction Method of the Boundary Value Problem of Composite Spherical Bessel Equations." American Journal of Mathematical Analysis, vol. 3, no. 2 (2015): 26-31. doi: 10.12691/ajma-3-2-1.

\section{Introduction}

With the rapid development of science and technology, a lot of edge sciences are produced, and a lot of new-style differential equations are appeared; differential equation is also extending in chemistry and biology at the same time, and a lot of reaction diffusion equations are appeared; differential equation is also widely used in materials science $[1,2]$, especially the composite material is widely used in industrial area, and some mathematical models of the composite material can be summed up in solving boundary value problem of composite differential equations, and which plays an important role in practical application.

In spherical coordinate system, Spherical Bessel equation is ordinary differential equation of radial component which is obtained by using the method of separation of variables to solve Laplace equation [3], it is common in well potential problem of the spherical square [4], its solution is called spherical Bessel function, and it is used to solve definite solution problem of heat conduction for spherically symmetric object $[5,6]$, therefore solving the boundary value problem of spherical Bessel equation is of significant.

About the research of solution structure for the boundary value problem of differential equation, in 2004, similar construction theory of solution was put forward in reference [7], namely expression of solution for the boundary value problem of differential equation has the similar structure, in other words, it is expressed by the form of continued fraction, and this theory was demonstrated in many references [8-14]. Such as boundary value problem of homogeneous linear differential equations of second order; boundary value problem of Euler hyper-geometric equation and so on.

This paper studied the following the boundary value problem of composite spherical Bessel equations:

$$
\left\{\begin{array}{l}
x^{2} z_{1}^{\prime \prime}+2 x z_{1}^{\prime}+\left[x^{2}-n_{1}\left(n_{1}+1\right)\right] z_{1}=0(a<x<c) \\
x^{2} z_{2}^{\prime \prime}+2 x z_{2}^{\prime}+\left[x^{2}-n_{2}\left(n_{2}+1\right)\right] z_{2}=0(c<x<b) \\
{\left[M z_{1}+(1+M N) z_{1}^{\prime}\right]_{x=a}=E} \\
\left.z_{1}\right|_{x=c}=\left.\left.\lambda z_{2}\right|_{x=c} z_{1}^{\prime}\right|_{x=c}=\left.\mu z_{2}^{\prime}\right|_{x=c} \\
{\left[P z_{2}+Q z_{2}^{\prime}\right]_{x=b}=0}
\end{array}\right.
$$

Where: $a, b, c, E, M, N, P, Q, \lambda, \mu$ are real constant, $n_{i}(i=1,2)$ is positive constant including zero, and $E \neq 0, P^{2}+Q^{2} \neq 0,0<a<c<b$.

Aimed at the boundary value problem (1), in part one, three lemmas are given; in part two, similar structure theorem of solution is demonstrated, similar kernel function in the left and right region is introduced, and two corollaries are obtained; in part three, the concrete step of similar construction method is given; finally, superiority of similar construction method is illuminated by giving a example. 


\section{Preliminary Knowledge}

Lemma 1. [15] spherical Bessel equation

$$
x^{2} y^{\prime \prime}+2 x y^{\prime}+\left[x^{2}-n(n+1)\right] y=0
$$

can be converted into the normal Bessel equation of $n+\frac{1}{2}$ order, namely

$$
x^{2} \frac{d^{2} \xi}{d x^{2}}+x \frac{d \xi}{d x}+\left[x^{2}-\left(n+\frac{1}{2}\right)^{2}\right] \xi=0
$$

Proof: $x^{2} y^{\prime \prime}+2 x y^{\prime}+\left[x^{2}-n(n+1)\right] y=0$ is converted into the equation $x^{2} \frac{d^{2} \xi}{d x^{2}}+x \frac{d \xi}{d x}+\left[x^{2}-\left(n+\frac{1}{2}\right)^{2}\right] \xi=0$ by variable substitution $y=\sqrt{\frac{\pi}{2 x}} \xi$.

Lemma 2. the general solution of spherical Bessel equation is

$$
y=\sqrt{\frac{\pi}{2 x}}\left[C_{1} Y_{n+\frac{1}{2}}(x)+C_{2} J_{n+\frac{1}{2}}(x)\right]
$$

Where: $C_{1}, C_{2}$ are any constant, $J_{n+\frac{1}{2}}(),. Y_{n+\frac{1}{2}}($.$) are$ first and second class Bessel function of $n+\frac{1}{2}$ order respective.

Proof: Because the general solution [16] of the normal Bessel equation (3) is

$$
\begin{gathered}
\xi=C_{1} Y_{n+\frac{1}{2}}(x)+C_{2} J_{n+\frac{1}{2}}(x) . \\
y=\sqrt{\frac{\pi}{2 x}} \xi=\sqrt{\frac{\pi}{2 x}}\left[C_{1} Y_{n+\frac{1}{2}}(x)+C_{2} J_{n+\frac{1}{2}}(x)\right]
\end{gathered}
$$

obtained by variable substitution $y=\sqrt{\frac{\pi}{2 x}} \xi$.

Lemma 3. function of two variables is structured, namely

$$
\psi_{m, n}(x, y)=Y_{m}(x) J_{n}(y)-J_{m}(x) Y_{n}(y)
$$

Where: $m, n$ are constant, $J_{v}(\cdot), Y_{v}(\cdot)$ are first and second Bessel function of $v$ order respective.

Therefore the following formula is easily obtained:

$$
\begin{gathered}
\frac{\partial}{\partial x} \psi_{v, v}(x, y)=\frac{v}{x} \psi_{v, v}(x, y)-\psi_{v+1, v}(x, y) \\
\frac{\partial}{\partial y} \psi_{v, v}(x, y)=\frac{v}{y} \psi_{v, v}(x, y)-\psi_{v, v+1}(x, y) \\
\frac{\partial^{2}}{\partial x \partial y} \psi_{v, v}(x, y)=\frac{\partial^{2}}{\partial y \partial x} \psi_{v, v}(x, y) \\
=\frac{v^{2}}{x y} \psi_{v, v}(x, y)-\frac{v}{x} \psi_{v, v+1}(x, y)-\frac{v}{y} \psi_{v+1, v}(x, y, t) \\
+\psi_{v+1, v+1}(x, y)
\end{gathered}
$$

Proof: According to the differential property of Bessel function [16]:

$$
\begin{aligned}
& \frac{d}{d t} J_{v}(t)=\frac{v}{t} J_{v}(t)-J_{v+1}(t), \\
& \frac{d}{d t} Y_{v}(t)=\frac{v}{t} Y_{v}(t)-Y_{v+1}(t) .
\end{aligned}
$$

We can obtain

$$
\begin{aligned}
& \frac{\partial}{\partial x} \psi_{v, v}(x, y)=\frac{d}{d x}\left[Y_{v}(x) J_{v}(y)-J_{v}(x) Y_{v}(y)\right] \\
& =\left[\frac{v}{x} Y_{v}(x)-Y_{v+1}(x)\right] J_{v}(y)-\left[\frac{v}{x} J_{v}(x)-J_{v+1}(x)\right] Y_{v}(y) \\
& =\frac{v}{x} \psi_{v, v}(x, y)-\psi_{v+1, v}(x, y)
\end{aligned}
$$

By a similar way, (7) and (8) can be demonstrated.

The guide functions are structured by two linearly independent solutions $\sqrt{\frac{\pi}{2 x}} Y_{n_{i}+\frac{1}{2}}(x), \sqrt{\frac{\pi}{2 x}} J{ }_{n_{i}+\frac{1}{2}}(x)$ of spherical Bessel equation, namely

$$
\begin{aligned}
& \phi_{0,0}^{i}(x, \xi)=\sqrt{\frac{\pi}{2 x}} Y_{n_{i}+\frac{1}{2}}(x) \cdot \sqrt{\frac{\pi}{2 \xi}} J_{n_{i}+\frac{1}{2}}(\xi) \\
& -\sqrt{\frac{\pi}{2 x}} J_{n_{i}+\frac{1}{2}}(x) \cdot \sqrt{\frac{\pi}{2 \xi}} Y_{n_{i}+\frac{1}{2}}(\xi) \\
& =\frac{\pi}{2} \sqrt{\frac{1}{x \xi}} \psi_{n_{i}+\frac{1}{2}, n_{i}+\frac{1}{2}}(x, \xi) \\
& \phi_{1,0}^{i}(x, \xi)=\frac{\partial}{\partial x} \phi_{0,0}^{i}(x, \xi) \\
& =\frac{\pi}{2} \sqrt{\frac{1}{x \xi}}\left[\frac{n_{i}}{x} \psi_{n_{i}+\frac{1}{2}, n_{i}+\frac{1}{2}}(x, \xi)-\psi_{n_{i}+\frac{3}{2}, n_{i}+\frac{1}{2}}(x, \xi)\right] \\
& \phi_{0,1}^{i}(x, \xi)=\frac{\partial}{\partial \xi} \phi_{0,0}^{i}(x, \xi) \\
& =\frac{\pi}{2} \sqrt{\frac{1}{x \xi}}\left[\frac{n_{i}}{\xi} \psi_{n_{i}+\frac{1}{2}, n_{i}+\frac{1}{2}}(x, \xi)-\psi_{n_{i}+\frac{1}{2}, n_{i}+\frac{3}{2}}(x, \xi)\right] \\
& \phi_{1,1}^{i}(x, \xi)=\frac{\partial^{2}}{\partial x \partial \xi} \phi_{0,0}^{i}(x, \xi)=\frac{\partial^{2}}{\partial \xi \partial x} \phi_{0,0}^{i}(x, \xi) \\
& =\frac{\pi n_{i}^{2}}{2} \sqrt{\frac{1}{x \xi}} \frac{1}{x \xi} \psi_{n_{i}+\frac{1}{2}, n_{i}+\frac{1}{2}}(x, \xi) \\
& -\frac{\pi n_{i}}{2} \sqrt{\frac{1}{x \xi}} \frac{1}{x} \psi_{n_{i}+\frac{1}{2}, n_{i}+\frac{3}{2}}(x, \xi) \\
& -\frac{\pi n_{i}}{2} \sqrt{\frac{1}{x \xi}} \frac{1}{\xi} \psi_{n_{i}+\frac{3}{2}, n_{i}+\frac{1}{2}}(x, \xi) \\
& +\frac{\pi}{2} \sqrt{\frac{1}{x \xi}} \psi_{n_{i}+\frac{3}{2}, n_{i}+\frac{3}{2}}(x, \xi)
\end{aligned}
$$

Where: $i=1,2$.

\section{The Main Theorem and its Proof}

Theorem If the boundary value problem (1) of composite spherical Bessel equations has unique solution, its solution in the left region $(a<x<c)$ is 


$$
z_{1}=E \cdot \frac{1}{M+\frac{1}{N+\varphi(a)}} \cdot \frac{1}{N+\varphi(a)} \cdot \varphi(x)
$$

its solution in the right region $(c<x<b)$ is

$$
\begin{gathered}
z_{2}=E \cdot \frac{1}{M+\frac{1}{N+\varphi(a)}} \cdot \frac{1}{N+\varphi(a)} \\
\cdot \frac{\phi_{0,1}^{1}(c, c)}{\lambda \varphi^{*}(c) \phi_{1,1}^{1}(a, c)-\mu \phi_{1,0}^{1}(a, c)} \cdot \varphi^{*}(x)
\end{gathered}
$$

Where: $\varphi(x)$ is called similar kernel function in the left region, namely

$$
\varphi(x)=\frac{\lambda \varphi^{*}(c) \phi_{0,1}^{1}(x, c)-\mu \phi_{0,0}^{1}(x, c)}{\lambda \varphi^{*}(c) \phi_{1,1}^{1}(a, c)-\mu \phi_{1,0}^{1}(a, c)},(a<x<c)
$$

$\varphi^{*}(x)$ is called similar kernel function in the right region, namely

$$
\varphi^{*}(x)=\frac{P \phi_{0,0}^{2}(x, b)+Q \phi_{0,1}^{2}(x, b)}{P \phi_{1,0}^{2}(c, b)+Q \phi_{1,1}^{2}(c, b)},(c<x<b)
$$

Proof: According to lemma 2, the general solution of the definite solution equation for the boundary value problem (1) is

$$
z_{i}(x)=C_{i}^{1} \sqrt{\frac{\pi}{2 x}} Y_{n_{i}+\frac{1}{2}}(x)+C_{i}^{2} \sqrt{\frac{\pi}{2 x}} J J_{n_{i}+\frac{1}{2}}(x),(i=1,2)(
$$

Where: $C_{i}^{1}, C_{i}^{2}(i=1,2)$ are any constant; when $i=1$, it indicates left region $(a<x<c)$; when $i=2$, it indicates right region $(c<x<b)$.

Taking the derivative of $z_{i}(x)$, namely

$$
\begin{aligned}
& z_{i}^{\prime}(x)=\frac{d}{d x}\left[C_{i}^{1} \sqrt{\frac{\pi}{2 x}} Y_{n_{i}+\frac{1}{2}}(x)+C_{i}^{2} \sqrt{\frac{\pi}{2 x}} J_{n_{i}+\frac{1}{2}}(x)\right] \\
& =\sqrt{\frac{\pi}{2}} x^{-\frac{3}{2}} n_{i}\left[C_{i}^{1} Y_{n_{i}+\frac{1}{2}}(x)+C_{i}^{2} J_{n_{i}+\frac{1}{2}}(x)\right] \\
& -\sqrt{\frac{\pi}{2 x}}\left[C_{i}^{1} Y_{n_{i}+\frac{3}{2}}(x)+C_{i}^{2} J_{n_{i}+\frac{3}{2}}(x)\right]
\end{aligned}
$$

The following formulas are obtained by putting the formula (17) plug into the boundary condition of the boundary value problem (1).

$$
\left.\begin{array}{l}
{\left[\begin{array}{l}
\left.\left(M \sqrt{\frac{\pi}{2 a}}+(1+M N) \sqrt{\frac{\pi}{2}} a^{-\frac{3}{2}} n_{1}\right) Y_{n_{1}+\frac{1}{2}}(a)\right] C_{1}^{1}+ \\
-(1+M N) \sqrt{\frac{\pi}{2 a}} Y n_{n_{1}+\frac{3}{2}}(a)
\end{array}\right]} \\
{\left[\left(M \sqrt{\frac{\pi}{2 a}}+(1+M N) \sqrt{\frac{\pi}{2}} a^{-\frac{3}{2}} n_{1}\right) J_{n_{1}+\frac{1}{2}}(a)\right] C_{1}^{2}=E} \\
-(1+M N) \sqrt{\frac{\pi}{2 a}} J_{n_{1}+\frac{3}{2}}(a)
\end{array}\right]
$$

$$
\begin{aligned}
& \sqrt{\frac{\pi}{2 c}} Y_{n_{1+\frac{1}{2}}}(c) C_{1}^{1}+\sqrt{\frac{\pi}{2 c}} J J_{n_{1}+\frac{1}{2}}(c) C_{1}^{2} \\
& -\lambda \sqrt{\frac{\pi}{2 c}} Y_{n_{2}+\frac{1}{2}}(c) C_{2}^{1}-\lambda \sqrt{\frac{\pi}{2 c}} J_{n_{2}+\frac{1}{2}}(c) C_{2}^{2}=0 \\
& {\left[\sqrt{\frac{\pi}{2}} c^{-\frac{3}{2}} n_{1} Y_{n_{1}+\frac{1}{2}}(c)-\sqrt{\frac{\pi}{2 c}} Y_{n_{1}+\frac{3}{2}}(c)\right] C_{1}^{1}} \\
& +\left[\sqrt{\frac{\pi}{2}} c^{-\frac{3}{2}} n_{1} J_{n_{1}+\frac{1}{2}}(c)-\sqrt{\frac{\pi}{2 c}} J_{n_{1}+\frac{3}{2}}(c)\right] C_{1}^{2}- \\
& {\left[\mu \sqrt{\frac{\pi}{2}} c^{-\frac{3}{2}} n_{2} Y_{n_{2}+\frac{1}{2}}(c)-\mu \sqrt{\frac{\pi}{2 c}} Y_{n_{2}+\frac{3}{2}}(c)\right] C_{2}^{1}} \\
& -\left[\mu \sqrt{\frac{\pi}{2}} c^{-\frac{3}{2}} n_{2} J_{n_{2}+\frac{1}{2}}(c)-\mu \sqrt{\frac{\pi}{2 c}} J_{n_{2}+\frac{3}{2}}(c)\right] C_{2}^{2}=0 \\
& {\left[\begin{array}{l}
P \sqrt{\frac{\pi}{2 b}} Y_{n_{2}+\frac{1}{2}}(b)+Q \sqrt{\frac{\pi}{2}} b^{-\frac{3}{2}} n_{2} Y_{n_{2}+\frac{1}{2}}(b) \\
-Q \sqrt{\frac{\pi}{2 b}} Y_{n_{2+\frac{3}{2}}}(b)
\end{array}\right] C_{2}^{1+}} \\
& {\left[\begin{array}{l}
P \sqrt{\frac{\pi}{2 b}} J_{n_{2+\frac{1}{2}}}(b)+Q \sqrt{\frac{\pi}{2}} b^{-\frac{3}{2}} n_{2} J_{n_{2}+\frac{1}{2}}(b) \\
-Q \sqrt{\frac{\pi}{2 b}} J_{n_{2+\frac{3}{2}}}(b)
\end{array}\right] C_{2}^{2}=0}
\end{aligned}
$$

Because the boundary value problem (1) has unique solution, determinant $\Delta$ of coefficients $C_{1}^{1}, C_{1}^{2}, C_{2}^{1}, C_{2}^{2}$ for equations (18)-(21) is not equal to zero, according to formulas (9)-(12), we can obtain

$$
\begin{aligned}
\Delta & =\lambda\left[P \phi_{0,0}^{2}(c, b)+Q \phi_{0,1}^{2}(c, b)\right] \\
& \cdot\left[M \phi_{0,1}^{1}(a, c)+(1+M N) \phi_{1,1}^{1}(a, c)\right] \\
& -\mu\left[P \phi_{1,0}^{2}(c, b)+Q \phi_{1,1}^{2}(c, b)\right] \\
& \cdot\left[M \phi_{0,0}^{1}(a, c)+(1+M N) \phi_{1,0}^{1}(a, c)\right]
\end{aligned}
$$

and then according to grammer rule, we can obtain value of coefficients $C_{1}^{1}, C_{1}^{2}, C_{2}^{1}, C_{2}^{2}$, namely

$$
\begin{aligned}
& C_{1}^{1}=\frac{E}{\Delta}\left\{\begin{array}{l}
\lambda\left[P \phi_{0,0}^{2}(c, b)+Q \phi_{0,1}^{2}(c, b)\right] \\
{\left[\sqrt{\frac{\pi}{2 c}}\left(\frac{n_{1}}{c} J_{n_{1}+\frac{1}{2}}(c)-J_{n_{1}+\frac{3}{2}}(c)\right)\right]}
\end{array}\right. \\
& \left.-\mu\left[P \phi_{1,0}^{2}(c, b)+Q \phi_{1,1}^{2}(c, b)\right] \sqrt{\frac{\pi}{2 c}} J_{n_{1}+\frac{1}{2}}(c)\right\} \\
& C_{1}^{2}=\frac{-E}{\Delta}\left\{\begin{array}{l}
\lambda\left[P \phi_{0,0}^{2}(c, b)+Q \phi_{0,1}^{2}(c, b)\right] \\
{\left[\sqrt{\frac{\pi}{2 c}}\left(\begin{array}{l}
\left.\frac{n_{1}}{c} Y_{n_{1}+\frac{1}{2}}(c)\right) \\
\left.-Y_{n_{1}+\frac{3}{2}}(c)\right)
\end{array}\right)\right]}
\end{array}\right. \\
& \left.-\mu\left[P \phi_{1,0}^{2}(c, b)+Q \phi_{1,1}^{2}(c, b)\right] \sqrt{\frac{\pi}{2 c}} Y_{n_{1}+\frac{1}{2}}(c)\right\}
\end{aligned}
$$




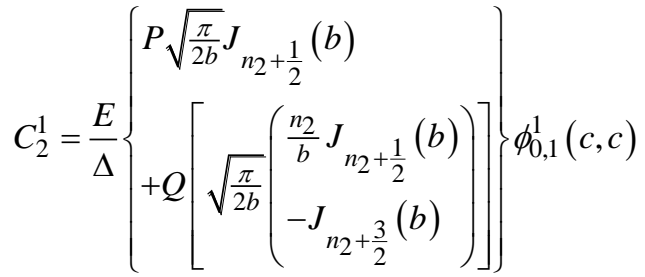

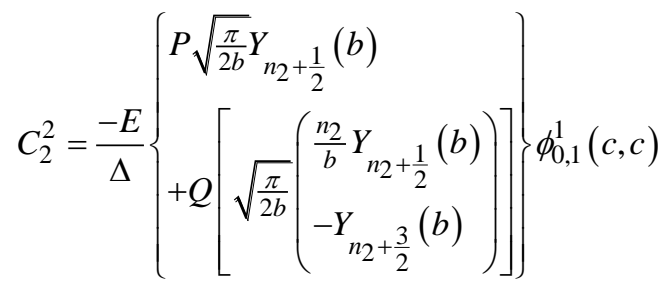

By putting the formula (23)-(26) plug into the formula (17) and using the similar kernel function in the left and right region (15);(16), the solution in the left and right region of boundary value problem (1) is obtained, namely (13);(14).

According to the theorem, it is easy to obtain the following corollary.

Corollary 1. If right boundary value condition of the boundary value problem (1) is $z_{2}(b)=0$, namely $Q=0, \quad P \neq 0$, the corresponding similar kernel function in the right region is

$$
\varphi^{*}(x)=\frac{\phi_{0,0}^{2}(x, b)}{\phi_{1,0}^{2}(c, b)} .
$$

Corollary 2. If right boundary value condition of the boundary value problem (1) is $z_{2}^{\prime}(b)=0$, namely $P=0, Q \neq 0$, the corresponding similar kernel function in the right region is

$$
\varphi^{*}(x)=\frac{\phi_{0,1}^{2}(x, b)}{\phi_{1,1}^{2}(c, b)}
$$

\section{Step of Similar Construction Method}

According to above the solving process for the boundary value problem (1), by analysis and summary, it is easy to obtain concrete step of similar construction method, which solving the boundary value (1).

Step 1. The function of two variables (5) is constructed, and then the guide functions (9)-(12) are constructed by using the two linearly independent solutions $\sqrt{\frac{\pi}{2 x}} Y_{n_{i}+\frac{1}{2}}(x) \sqrt{\frac{\pi}{2 x}} J{ }_{n_{i}+\frac{1}{2}}(x)(i=1.2)$ of the definite solution equation for the boundary value problem (1).

Step 2. According to the guide functions in the right region $\phi_{0,0}^{2}(x, \xi) 、 \phi_{0,1}^{2}(x, \xi) 、 \phi_{1,0}^{2}(x, \xi) 、 \phi_{1,1}^{2}(x, \xi)$ and the coefficients $P, Q$ of the right boundary value condition $\left[P z_{2}+Q z_{2}^{\prime}\right]_{x=b}=0$, the similar kernel function in the right region $\varphi^{*}(x)$ is assembled by using the formula (16); According to the guide functions in the left region $\phi_{0,0}^{1}(x, \xi) 、 \phi_{0,1}^{1}(x, \xi) 、 \phi_{1,0}^{1}(x, \xi) 、 \phi_{1,1}^{1}(x, \xi) \quad ; \quad \varphi^{*}(c)$ and the coefficients $\lambda, \mu$ of the connective condition $\left.z_{1}\right|_{x=c}=\left.\lambda z_{2}\right|_{x=c},\left.z_{1}^{\prime}\right|_{x=c}=\left.\mu z_{2}^{\prime}\right|_{x=c}$, the similar kernel function in the left region $\varphi(x)$ is assembled by using the formula (15).

Step 3. According to the coefficients $E 、 M 、 N$ of the left boundary value condition $\left[M z_{1}+(1+M N) z_{1}^{\prime}\right]_{x=a}=E$ and $\varphi(x), \varphi(a)$, the solution in the left region of the boundary value problem (1) is assembled by using the formula (13); According to the coefficients $E 、 M 、 N$ of the left boundary value condition $\left[M z_{1}+(1+M N) z_{1}^{\prime}\right]_{x=a}=E$ and $\varphi(a)$, the guide functions in the left region $\phi_{0,1}^{1}(x, \xi) 、 \phi_{1,0}^{1}(x, \xi) 、 \phi_{1,1}^{1}(x, \xi)$, the coefficients $\lambda 、 \mu$ of joint conditions $\left.z_{1}\right|_{x=c}=\left.\lambda z_{2}\right|_{x=c},\left.z_{1}^{\prime}\right|_{x=c}=\left.\mu z_{2}^{\prime}\right|_{x=c}$ and the similar kernel function in the right region $\varphi^{*}(x)$, the solution in the right region is assembled by using the formula (14) for the boundary value problem (1).

\section{Example}

The following boundary value problem is solved by using the above step of similar construction method.

$$
\left\{\begin{array}{l}
x^{2} z_{1}^{\prime \prime}+2 x z_{1}^{\prime}+\left(x^{2}-2\right) z_{1}=0(1<x<10) \\
x^{2} z_{2}^{\prime \prime}+2 x z_{2}^{\prime}+\left(x^{2}-6\right) z_{2}=0(10<x<20) \\
\left(z_{1}+2 z_{1}^{\prime}\right)_{x=1}=1 \\
\left.z_{1}\right|_{x=10}=\left.z_{2}\right|_{x=10} \\
\left.z_{1}^{\prime}\right|_{x=10}=\left.z_{2}^{\prime}\right|_{x=10} \\
\left(z_{2}+z_{2}^{\prime}\right)_{x=20}=0
\end{array}\right.
$$

Where: In this case $a=1, b=20, c=10, M=N=E=P=Q=\lambda=\mu=1$, $n_{1}=1, n_{2}=2$.

Step 1. The function of two variables and guide functions are constructed, namely $\psi_{\frac{3}{2}, \frac{3}{2}}(x, \xi)=Y_{\frac{3}{2}}(x) J_{\frac{3}{2}}(\xi)-J_{\frac{3}{2}}(x) Y_{\frac{3}{2}}(\xi) \quad$ and $\psi_{\frac{5}{2}, \frac{5}{2}}(x, \xi)=Y_{\frac{5}{2}}(x) J_{\frac{5}{2}}(\xi)-J_{\frac{5}{2}}(x) Y_{\frac{5}{2}}(\xi) \quad$ are $\quad$ called function of two variables, the following functions are called guide function.

$$
\begin{aligned}
\phi_{0,0}^{1}(x, \xi) & =\sqrt{\frac{\pi}{2 x}} Y_{\frac{3}{2}}(x) \cdot \sqrt{\frac{\pi}{2 \xi}} J_{\frac{3}{2}}(\xi) \\
& -\sqrt{\frac{\pi}{2 x}} J_{\frac{3}{2}}(x) \cdot \sqrt{\frac{\pi}{2 \xi}} Y_{\frac{3}{2}}(\xi) \\
& =\frac{\pi}{2} \sqrt{\frac{1}{x \xi}} \psi_{\frac{3}{2}, \frac{3}{2}}(x, \xi)
\end{aligned}
$$




$$
\begin{aligned}
& \phi_{0,0}^{2}(x, \xi)=\sqrt{\frac{\pi}{2 x}} Y_{\frac{5}{2}}(x) \cdot \sqrt{\frac{\pi}{2 \xi}} J_{\frac{5}{2}}(\xi) \\
& -\sqrt{\frac{\pi}{2 x}} J_{\frac{5}{2}}(x) \cdot \sqrt{\frac{\pi}{2 \xi}} Y_{\frac{5}{2}}(\xi) \\
& =\frac{\pi}{2} \sqrt{\frac{1}{x \xi}} \psi_{\frac{5}{2}, \frac{5}{2}}(x, \xi) \text {, } \\
& \phi_{1,0}^{1}(x, \xi)=\frac{\partial}{\partial x} \phi_{0,0}^{1}(x, \xi) \\
& =\frac{\pi}{2} \sqrt{\frac{1}{x \xi}}\left[\frac{1}{x} \psi_{\frac{3}{2}, \frac{3}{2}}(x, \xi)-\psi_{\frac{5}{2}, \frac{3}{2}}(x, \xi)\right] \\
& \phi_{1,0}^{2}(x, \xi)=\frac{\partial}{\partial x} \phi_{0,0}^{2}(x, \xi) \\
& =\frac{\pi}{2} \sqrt{\frac{1}{x \xi}}\left[\frac{2}{x} \psi_{\frac{5}{2}, \frac{5}{2}}(x, \xi)-\psi_{\frac{7}{2}, \frac{5}{2}}(x, \xi)\right] \\
& \phi_{0,1}^{1}(x, \xi)=\frac{\partial}{\partial \xi} \phi_{0,0}^{1}(x, \xi) \\
& =\frac{\pi}{2} \sqrt{\frac{1}{x \xi}}\left[\frac{1}{\xi} \psi_{\frac{3}{2}, \frac{3}{2}}(x, \xi)-\psi_{\frac{3}{2}, \frac{5}{2}}(x, \xi)\right] \\
& \phi_{0,1}^{2}(x, \xi)=\frac{\partial}{\partial \xi} \phi_{0,0}^{2}(x, \xi) \\
& =\frac{\pi}{2} \sqrt{\frac{1}{x \xi}}\left[\frac{2}{\xi} \psi_{\frac{5}{2}, \frac{5}{2}}(x, \xi)-\psi_{\frac{5}{2}, \frac{7}{2}}(x, \xi)\right] \\
& \phi_{1,1}^{1}(x, \xi)=\frac{\partial^{2}}{\partial x \partial \xi} \phi_{0,0}^{1}(x, \xi)=\frac{\partial^{2}}{\partial \xi \partial x} \phi_{0,0}^{1}(x, \xi) \\
& =\frac{\pi}{2} \sqrt{\frac{1}{x \xi}} \frac{1}{x \xi} \psi_{\frac{3}{2}, \frac{3}{2}}(x, \xi) \\
& -\frac{\pi}{2} \sqrt{\frac{1}{x \xi}} \frac{1}{x} \psi_{\frac{3}{2}, \frac{5}{2}}(x, \xi) \\
& -\frac{\pi}{2} \sqrt{\frac{1}{x \xi}} \frac{1}{\xi} \psi_{\frac{5}{2}, \frac{3}{2}}(x, \xi) \\
& +\frac{\pi}{2} \sqrt{\frac{1}{x \xi}} \psi_{\frac{5}{2}, \frac{5}{2}}(x, \xi) \\
& \phi_{1,1}^{2}(x, \xi)=\frac{\partial^{2}}{\partial x \partial \xi} \phi_{0,0}^{2}(x, \xi)=\frac{\partial^{2}}{\partial \xi \partial x} \phi_{0,0}^{2}(x, \xi) \\
& =2 \pi \sqrt{\frac{1}{x \xi}} \frac{1}{x \xi} \psi_{\frac{5}{2}, \frac{5}{2}}(x, \xi) \\
& -\pi \sqrt{\frac{1}{x \xi}} \frac{1}{x} \psi_{\frac{5}{2}, \frac{7}{2}}(x, \xi) \\
& -\pi \sqrt{\frac{1}{x \xi}} \frac{1}{\xi} \psi_{\frac{7}{2}, \frac{5}{2}}(x, \xi) \\
& +\frac{\pi}{2} \sqrt{\frac{1}{x \xi}} \psi_{\frac{7}{2}, \frac{7}{2}}(x, \xi)
\end{aligned}
$$

Step 2. The similar kernel functions are constructed, namely $\quad \varphi^{*}(x)=\frac{\phi_{0,0}^{2}(x, 20)+\phi_{0,1}^{2}(x, 20)}{\phi_{1,0}^{2}(10,20)+\phi_{1,1}^{2}(10,20)} \quad$ is $\quad$ called similar kernel function in the right region of the boundary value problem (27).

$$
\varphi(x)=\frac{\varphi^{*}(10) \phi_{0,1}^{1}(x, 10)-\phi_{0,0}^{1}(x, 10)}{\varphi^{*}(10) \phi_{1,1}^{1}(1,10)-\phi_{1,0}^{1}(1,10)} \text { is called similar }
$$

kernel in the left region of the boundary value problem (27) and $\varphi(1)=\frac{\varphi^{*}(10) \phi_{0,1}^{1}(1,10)-\phi_{0,0}^{1}(1,10)}{\varphi^{*}(10) \phi_{1,1}^{1}(1,10)-\phi_{1,0}^{1}(1,10)}$.

Step 3. The solution is constructed, namely $z_{1}=\frac{1}{1+\frac{1}{1+\varphi(1)}} \cdot \frac{1}{1+\varphi(1)} \cdot \varphi(x) \quad(1<x<10)$ is called solution in the left region of the boundary value problem (27).

$$
\begin{aligned}
& z_{2}=\frac{1}{1+\frac{1}{1+\varphi(1)}} \cdot \frac{1}{1+\varphi(1)} \\
& \cdot \frac{\phi_{0,1}^{1}(10,10)}{\varphi^{*}(10) \phi_{1,1}^{1}(1,10)-\phi_{1,0}^{1}(1,10)} \cdot \varphi^{*}(x),(10<x<20)
\end{aligned}
$$

called solution in the right region of the boundary value problem (27).

According to the concrete step of similar construction method, the changing curve of the solution in the designated area is drawn by using Matlab for the boundary value problem (27), namely figure 1

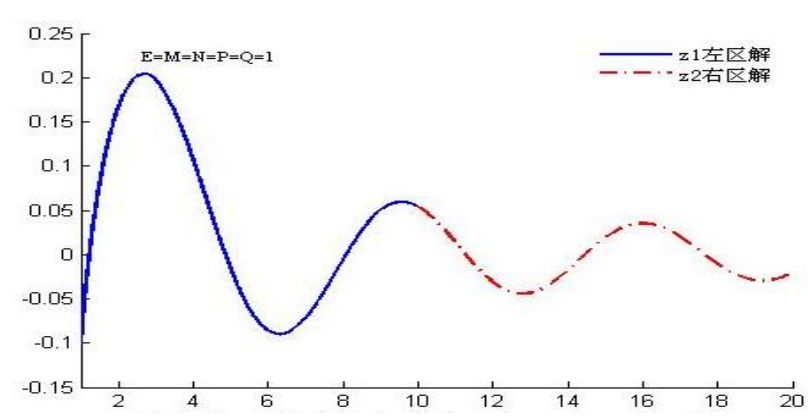

Figure 1. Curve of solution to the boundary value problem (27)

\section{Conclusion}

(1) Aimed at the solution (13) and (14) of the boundary value problem (1), it is expressed by continued fraction in form, namely similar structure.

(2) For solving process of the boundary value problem (1), similar construction method that is put forward can solve its solution, and this method is simple and effective, the step of this method plays an important role in drawing the changing curve of solution of the corresponding boundary value problem for men of mathematics.

(3) For the boundary value problem (1), when the coefficient of right boundary value condition changes, it is merely changing similar kernel function, but expression form of the solution is not changing.

(4) Observing the formula (13) and (14), the following relational expression is obtained, namely $\left[z_{1}(x)+N z_{1}^{\prime}(x)\right]_{x=a}=\frac{E}{M+\frac{1}{N+\varphi(a)}}$. 


\section{Acknowledgment}

This Project is supported by the Scientific Research Fund of Sichuan Provincial Education Department of China under Grant No.12ZA164.

\section{References}

[1] Yiting Wang, Ping Wang, Xun Wang. The application of the differential equation in the studies of materials (in Chinese). Academy, 2011, 2 (4): 21-22.

[2] Xiufen Wang, Zhihong Ma, Zhimin Mu. Study of the temperature control material force deformation Based on the differential equation model (in Chinese). Coal Technology, 2010, 24 (5): 208210.

[3] Shanjie zhang. Vector analysis; cylindrical function and spherical function. Nanjing: Nanjing University Press, 2011.

[4] Yongde Zhang. Quantum mechanics. Beijing: Science Press, 2005.

[5] Ying Gao, Hanwei Yi. Sula Baiya, ect. Computation of energy levels of I2 molecule by spherical Bessel function expansion method (in Chinese). Journal of Changchun University of science and technology, 2009, 32 (3): 511-512.

[6] Xiaozeng Wang, Hui Zeng, Jiuhong Yang. Analysis on steel ball surface temperature change during heat treatment process (in Chinese). Hot Working Technology, 2012, 41 (4): 187-189 (-193).

[7] Shunchu Li, Minhui Jia. The Formal Similarity of Solutions on the Class of Differential Equation. Journal of UEST of China, 2004, 33 (Supp.): 95-98.
[8] Pengshe Zheng, Shunchu Li, Weibing Zhu. The similar structure of pressure distribution in the double porosity composite reservoir (in Chinese). Drilling \& Production Technology, 2008, 31 (4): 8081.

[9] Shunchu Li. The similar structure of solution to the boundary value problem for second-order linear homogeneous differential equations (in Chinese). Journal of Xihua University (Natural Science Edition), 2009, 28 (5): 40-41 (to 90).

[10] Shunchu Li. Preliminary exploration and prospects of the similar structure of solutions of differential equations (in Chinese). Journal of Xihua University (Natural Science Edition), 2010, 29 (2): 223-226 (to 238).

[11] Yibin Tian, Shunchu Li, Quanyong Li, etc. The similar structure of Tschebyscheff equation on boundary value problem (in Chinese). Sichuan Ordnance Journal, 2011, 32 (1): 155-156.

[12] Xuxia Xiao, Li Shunchu Li. The similar structure of solutions for the boundary value problem of Euler's Hypergeometric differential equation (in Chinese). Journal of Inner Mongolia Normal University (Natural Science Edition), 2012, 41 (6): 597$600(-603)$.

[13] Quanyong Li, Shunchu Li, Ke Li, etc. Study of the seepage model in dual-porosity composite media reservoir based on similar structure of solution (in Chinese). Drilling \& Production Technology, 2012, 35 (2): 54-56.

[14] Xitao Bao, Shunchu Li, ZhiJian Liao. Similar constructive method of solution for the boundary value problem of Euler Hypergeometric differential equation (in Chinese). Journal of Southwest University of Science and Technology, 2012, 27 (4): 101-105.

[15] Shunchu Li, Bingguang Huang. Laplace transform and Bessel functions and the theoretical basis of well test analysis. Beijing: Petroleum Industry Press, 2000.

[16] Shishi Liu, Shida Liu. Special function. Beijing: China Meteorological Press, 2002. 